\title{
Correspondence
}

Home assessments in old age psychiatry

Richardson \& Orrell (2002) confuse domiciliary and home assessment visits by consultants. It is important to be clear about the distinction between them.

Domiciliary visits incur additional payment and are defined as occurring at the request of the general practitioner, and normally in his/her company, to advise on the diagnosis or treatment, where the patient cannot attend hospital on medical grounds.

Home assessment visits, or community clinics, are commonly carried out by old age psychiatrists within their contracted sessions and have been shown to have some advantages in comparison with traditional out-patient clinics: they are more likely to result in the patient being seen, are more flexible and may not be more time-consuming (Benbow, 1990).

When we used a detailed questionnaire to survey the workloads of old age psychiatrists, we asked about domiciliary visits, new home assessment visits and follow-up community clinic home visits (Jolley \& Benbow, 1997). Respondents reported all three activities. Consultant old age psychiatrists who were working with colleagues (i.e. not single-handed) spent more time on home assessment visits, saw more new patients on home visits and saw significantly more follow-up community clinic patients. Old age psychiatrists who worked alone spent more time on domiciliary visiting and saw more patients on domiciliary visits (Benbow \& Jolley, 1999). For community services there are advantages in grouping consultants together for mutual support.

We agree with Richardson \& Orrell (and with the National Service Framework for Older People (Department of Health, 2001)) that home care is part of mental health practice, but the emphasis of their paper appears to be on shifting assessments from the doctor to the team. Services work best when teams (including psychiatrists, nurses, social workers and others) work towards the common goal of patient care. The definition of a domiciliary visit is irrelevant when old age psychiatry community teams are properly resourced and structured.
Benbow, S. M. (1990) The community clinic - its advantages and disadvantages. International Journal of Geriatric Psychiatry, 2, 119-121.

— \& Jolley, D. J. (1999) Gender, isolation, work patterns and stress amongst old age psychiatrists. International Journal of Geriatric Psychiatry, 14, 719-725.

Department of Health (2001) National Service Framework for Older People. London: Department of Health.

Jolley, D. J. \& Benbow, S. M. (1997) The everyday work of geriatric psychiatrists. International Journal of Geriatric Psychiatry, 12, 109-113.

Richardson, B. \& Orrell, M. (2002) Home assessments in old age psychiatry. Advances in Psychiatric Treatment, 8, 59-65.

Susan M. Benbow Consultant Old Age Psychiatrist, David Jolley Professor of Old Age Psychiatry, Wolverhampton Health Care NHS Trust, Penn Hospital, Penn Road, Wolverhampton, West Midlands WV4 5HA, UK

\section{Solution-focused brief therapy}

I was interested to read the paper on solutionfocused brief therapy by Iveson (2002), and the commentary by Göpfert (2002).

Solution-focused brief therapy is a valuable treatment approach within psychiatry, although the outcome research shows that other approaches are needed for some patients.

Göpfert states that the use of solution-focused brief therapy is held back because the training culture is 'for-profit, private'. In fact, there has been an accredited training course at St Martin's College, Carlisle, for 5 years. There are college-based courses at Guildford and Canterbury and an MA in solutionfocused brief therapy at the University of Birmingham. None of these courses is based on private funding.

Training in other approaches to psychotherapy relies heavily on non-public funding from various sources. Training costs are significantly less in solution-focused brief therapy than in other psychotherapies.

Göpfert, M. (2002) Solution-focused brief therapy: Commentary. Advances in Psychiatric Treatment, 8, 157-157. Iveson, C. (2002) Solution-focused brief therapy. Advances in Psychiatric Treatment, 8, 149-156.

Alasdair J. Macdonald Honorary Consultant Psychiatrist, North Cumbria Mental Health and Learning Disabilities NHS Trust, Carleton Clinic, Cumwhinton Drive, Carlisle CA1 3SX, UK 